\title{
Diseño de una prótesis de mano para uso en teclados con interfaz sEMG.
}

\section{Design of a hand prosthesis for use of keyboards with sEMG interface}

\author{
Ernesto-Eduardo López-López \\ Facultad de Medicina \\ Universidad Autónoma del Estado de México, México \\ ernesto.lopez.lbm@outlook.com
}

\section{Rigoberto Martínez-Méndez}

Facultad de Ingeniería

Universidad Autónoma del Estado de México, México rigo@ieee.org

\section{Adriana H. Vilchis González}

Facultad de Ingeniería

Universidad Autónoma del Estado de México, México avilchisg@uaemex.mx 


\section{Resumen:}

Objetivos: En la actualidad existen diversas opciones de prótesis de mano, sin embargo, ninguna de ellas es adecuada para la utilización de teclados, debido a la lentitud o falta de movimientos adecuados. En este trabajo se plantea una prótesis de mano de bajo costo, controlada por señales electromiográficas, con diseño específico para el uso de teclados de computadora.

Metodología: Se analizaron los datos antropométricos de la población mexicana, los requerimientos de fuerza y las diferentes opciones de materiales, transmisión de movimientos y adquisición y análisis de señales EMG, para proponer una prótesis capaz de interactuar con teclados.

Resultados: Se presenta el diseño de una prótesis de mano para fabricarse por medio de impresión 3D, que cumple con las características de fuerza y velocidad necesarias como para mejorar la interfaz entre usuario y teclados de computadora.

Relevancia: Existen muchas prótesis, pero la mayoría son costosas y diseñadas para trabajos pesados o complejos que hacen a las prótesis lentas, pesadas y costosas. Aquí se presenta una opción para prótesis de mano de uso específico para el uso de teclados.

Palabras clave: Prótesis de mano, interfaz mioeléctrica, robótica.

\section{Abstract:}

Objectives: Nowadays there are several options of hand prostheses, however none of them is adequate for its use in keyboards, mainly due to their slow response and lack of necessary movements. This work describes the conceptual design of a low-cost hand prosthesis, controlled by electromyography signals and specialized for the use of keyboards.

Methodology: Anthropometric data of the Mexican and Latin-American population were analyzed as well as force requirements, materials available, movement transmission techniques, acquisition and analysis of electromyography signals, in order to propose a prosthesis design capable to interface with keyboards.

Results: Is presented a proposal of a hand prosthesis specialized for the use of keyboards. This prosthesis was designed to be developed using 3D printing technology. According with the design methodology, the prosthesis will be capable to comply with the force and speed necessary to improve the interface between amputee users and keyboards. 
Relevance: There exists many hand prosthesis, but most of them are designed for complex or heavy tasks, making the prosthesis slow, heavy and expensive. In this work is presented the mechanical and electronic design of an alternative prosthesis specialized for use of keyboards. This prosthesis could improve the quality of life of people which need to use keyboards during their daily life activities.

Keywords: hand prosthesis, myoelectric interface, robotics.

\section{Introducción}

Se estima que a nivel mundial existen al menos 10 millones de personas amputadas de las cuales el $30 \%$ son amputaciones de brazo y mano ( 3 millones) y 2.4 millones son personas viviendo en países en vías de desarrollo (Amputee Coalition, 2016; ISHN, 2014; LeBlanc, 2011). En México se realizan anualmente alrededor de 2300 amputaciones a nivel de muñeca y mano, de las cuales, el $85 \%$ son sufridas por hombres. Especialmente adultos entre 20 y 39 años (Secretaría del Trabajo y Previsión Social, 2010; Vazquez-Vela, 2015).

Por otro lado, si bien es cierto que existen opciones de prótesis para los pacientes amputados, éstas no son suficientes. Se estima que a nivel nacional, sólo se producen 1500 prótesis (incluyendo prótesis de piernas y brazos), esto solo alcanza a cubrir el $5.5 \%$ de los amputados anuales nacionales (VazquezVela, 2015) y no existen datos sobre cuántas de estas prótesis son de mano.

La otra opción que tienen los pacientes amputados son las prótesis comerciales fabricadas por empresas privadas. Sin embargo éstas son demasiado costosas para el mexicano promedio. Una búsqueda del estado del arte, limitado sólo a prótesis de mano, con control mioeléctrico, ya que es la propuesta de este trabajo, arrojó los datos que se muestran en la Tabla 1.

Tabla 1. Características de prótesis de mano con control mioeléctrico.

\begin{tabular}{|c|c|c|}
\hline Prótesis & Características & $\begin{array}{l}\text { Costo MXN/ } \\
\text { Disponibilidad }\end{array}$ \\
\hline ( Touch Bionics, 2015) & $\begin{array}{l}\text { - Rotación de muñeca de } 360^{\circ} \text {. } \\
\text { - Fuerza por actuador: } 5 \mathrm{Kg} \text {. } \\
\text { - Alimentación: } 7.4 \mathrm{~V} \\
\text { - } 36 \text { movimientos. } \\
\text { - Peso: } 1,590 \mathrm{~g} \\
\text { - } 15 \mathrm{DOF} \\
\text { - Diseño para gesto de pinza. Es capaz de interactuar } \\
\text { con un mouse. } \\
\text { - Solo responde a movimientos preprogramados }\end{array}$ & $\begin{array}{c}\$ 1,482,531 \text { a } \\
\$ 2,965,062 \\
\text { (Importación) }\end{array}$ \\
\hline
\end{tabular}




\begin{tabular}{|c|c|c|}
\hline $\begin{array}{l}\text { Michelangelo prosthetic } \\
\text { hand (Ottobock, 2019) }\end{array}$ & $\begin{array}{l}\text { - Flexión y rotación de muñeca en } 360 \text { - } \\
\text { - Cada actuador ejerce una fuerza de } 5 \mathrm{Kg} \text {. } \\
\text { - Alimentado con } 11 \mathrm{~V} \\
\text { - } 7 \text { movimientos. } \\
\text { - Peso: } 668 \mathrm{~g} \text {. } \\
\text { - } 16 \text { DOF } \\
\text { - Velocidad: } 32.5 \mathrm{~mm} / \mathrm{s} \\
\text { - Diseñado para realizar movimientos de apoyo para } \\
\text { manipulación de objetos. } \\
\text { - Solo responde a movimientos preprogramados }\end{array}$ & $\begin{array}{c}\$ 988,426 \text { a } \\
\$ 2,965,062 \\
\text { (Importación) }\end{array}$ \\
\hline $\begin{array}{c}\text { Bebionic } 3 \\
\text { (Bebionic, 2012) }\end{array}$ & $\begin{array}{l}\text { - Rotación de muñeca en } 360^{\circ} \\
\text { - Capaz de soportar } 90 \mathrm{Kg} \text {. } \\
\text { - } 14 \text { movimientos } \\
\text { - Alimentado con } 7.4 \mathrm{~V} \\
\text { - Peso: } 628 \mathrm{~g} \\
\text {-14 DOF } \\
\text { - Tiempo de apertura/cierre: } 0.5 \mathrm{~s} \\
\text { - Diseño para gesto de pinza, es capaz de ser } \\
\text { utilizado para interactuar con un mouse. } \\
\text { - Control mioeléctrico con movimientos programados. }\end{array}$ & $\begin{array}{c}\$ 473,840 \text { a } \\
\$ 671,776 \\
\text { (Importación) }\end{array}$ \\
\hline $\begin{array}{c}\text { Luke Arm } \\
\text { (Mobius Bionics, 2018) }\end{array}$ & $\begin{array}{l}\text { - Cuenta con } 10 \text { movimientos programables, } \\
\text { retroalimentación táctil. } \\
\text { - Peso: } 1,400 \mathrm{~g} \\
\text { - } 14 \text { DOF } \\
\text { - Diseño para gesto de pinza fino, y control a través } \\
\text { de sensores externos. } \\
\text { - Control mioléctrico y sensores de presión y } \\
\text { movimiento. }\end{array}$ & $\begin{array}{c}\$ 1,919,310 \\
\text { (No disponible) }\end{array}$ \\
\hline (Probionics, 2015) & $\begin{array}{l}\text { - Prótesis eléctricas, que utilizan el gesto de pinza. } \\
\text { - Alimentado a } 12 \mathrm{~V} \text {. } \\
\text { - } 2 \text { DOF } \\
\text { - Diseño para gesto de pinza. } \\
\text { - Control mioeléctrico con movimientos programados. }\end{array}$ & $\begin{array}{l}\$ 60,000 \text { a } \\
\$ 180,000 \\
\text { (México) }\end{array}$ \\
\hline $\begin{array}{l}\text { Youbionic arm } \\
\text { (Youbionic, 2014) }\end{array}$ & $\begin{array}{l}\text { - Brazo y mano impreso en 3D. } \\
\text { - Se vende con actuadores que ejercen hasta } 5 \mathrm{~kg} \text { de } \\
\text { fuerza. } \\
\text { - Sin uso específico. } \\
\text { - Control y tiempo de respuesta depende de la } \\
\text { construcción. }\end{array}$ & $\begin{array}{l}\$ 2,445.65 \\
\text { (Importación) }\end{array}$ \\
\hline
\end{tabular}




\begin{tabular}{|c|c|c|}
\hline $\begin{array}{c}\text { Azzurra Hand } \\
\text { (Prensilia s.r.l, 2017) }\end{array}$ & $\begin{array}{l}\text { - } 10 \text { articulaciones } \\
\text { - Al ser diseñado para proyectos, la cantidad de } \\
\text { movimientos, control, fuerza y peso total, dependen } \\
\text { del desarrollador. } \\
\text { - Peso: } 640 \mathrm{~g} \\
\text { - Sin uso específico. } \\
\text { - Control y tiempo de respuesta depende de la } \\
\text { construcción. }\end{array}$ & No disponible. \\
\hline $\begin{array}{c}\text { Hero Arm } \\
\text { (Open bionics, 2018) }\end{array}$ & $\begin{array}{l}\text { - En venta la prótesis como armazón. } \\
\text { - Al ser diseñado para proyectos, la cantidad de } \\
\text { movimientos, control, fuerza y peso total, dependen } \\
\text { del desarrollador. } \\
\text { - Peso: } 150 \mathrm{~g} \\
\text { - Sin un uso específico, capaz de realizar gesto de } \\
\text { pinza. } \\
\text { - Control y tiempo de respuesta depende de la } \\
\text { construcción. }\end{array}$ & $\begin{array}{c}\$ 2,942.70 \\
\text { (Importación) }\end{array}$ \\
\hline $\begin{array}{c}\text { MyoArm } \\
\text { (Perez-Rodriguez, 2017) }\end{array}$ & $\begin{array}{l}\text { - Prótesis desarrollada mediante brazalete Myo, sin } \\
\text { movimiento de muñeca. } \\
\text { - Con actuadores que ejercen hasta } 5 \mathrm{~kg} \text { de fuerza. } \\
\text { - Sin un uso específico, capaz de realizar gesto de } \\
\text { pinza. } \\
\text { - Control por redes neuronales. } \\
\text { - Tiempo de respuesta } 4-7 \text { segundos. } \\
\text { - Control mioeléctrico con movimientos programados. }\end{array}$ & $\begin{array}{c}\$ 10,000 \\
\text { No disponible }\end{array}$ \\
\hline $\begin{array}{l}\text { CIMAV-CONACYT } \\
\text { (CONACYT, 2018) }\end{array}$ & $\begin{array}{l}\text { - Prótesis desarrollada mediante brazalete Myo, sin } \\
\text { movimiento de muñeca. } \\
\text { - Con actuadores que ejercen hasta } 5 \mathrm{~kg} \text { de fuerza. } \\
\text { - Sin un uso específico, capaz de realizar gesto de } \\
\text { pinza. } \\
\text { - Control por redes neuronales. } \\
\text { - Tiempo de respuesta } 2-4 \text { segundos. } \\
\text { - Control mioeléctrico }\end{array}$ & $\begin{array}{c}\$ 10,000 \\
\text { No disponible }\end{array}$ \\
\hline
\end{tabular}

En la Tabla 1 se puede observar que la mayoría de las prótesis de mano disponibles tienen un alto costo, comparado con el ingreso promedio de los mexicanos, el cual ronda los 6 mil pesos mensuales (Vanguardia, 2018). Esto implicaría el salario íntegro de casi 10 meses para poder adquirir la prótesis comercial más económica (Probionics, 2015) y hasta 20 años para la prótesis 
más avanzada. Otras prótesis que se han reportado en la literatura, son prototipos, que no se encuentran a la venta y la mayoría están en fase de desarrollo aún. Un análisis más completo de prótesis de brazo y mano con control, no necesariamente por EMG, enfocado en prótesis con fabricación usando impresión 3D, reporta costos de prótesis de 500 USD (Kate, Smit, \& Breedveld, 2017).

Otra grave limitante es la lenta respuesta que la mayoría de las prótesis presentan en sus movimientos, especialmente aquellas que cuentan con muchos sensores o algoritmos complicados para la detección e interpretación de intención de movimiento. Por ejemplo, el prototipo diseñado por el Centro de Investigación en Materiales Avanzados, (CONACyT, 2018), utiliza 8 sensores de EMG y tarda casi medio segundo en reaccionar a los comandos y aún con algunos errores en la activación, como se puede observar en los videos publicados por los autores (https://www.youtube.com/watch?v=Euol7I_YRmU).

Otras prótesis, como la Bebionic (Bebionic, 2012), tarda hasta $0.5 \mathrm{~s}$ en cerrar o abrir la mano, lo que implica que son demasiado lentas como para activar las teclas si se deseara escribir en una computadora o jugar algún videojuego. En 2017 surgió la propuesta de una mano para tocar piano usando ultrasonido en lugar de EMG (Jason, 2017). Sí bien esta propuesta promete una mayor rapidez en la activación de los dedos protésicos, depende de un sistema de ultrasonido y equipo de cómputo externo para realizar el procesamiento de las señales. Lo anterior elimina toda portabilidad del sistema y aumenta su costo y complejidad impidiendo que se convierta en una alternativa viable a corto plazo para los usuarios comunes.

A la fecha no se ha reportado ninguna prótesis, ni comercial ni en fase de prototipo que esté diseñada para la interacción con teclados como la planteada en este documento. Los autores de este trabajo han considerado relevante diseñar y posteriormente desarrollar una prótesis de bajo costo para interactuar con teclados debido a que, ésta proporcionaría un mayor grado de autonomía al amputado. De esta manera el amputado podría, no solo realizar trabajos de oficina que requirieran el uso de computadoras, sino que también podría reducir su estrés jugando videojuegos o tocando instrumentos de teclas, como un piano, por ejemplo. En este trabajo se presenta el diseño de una prótesis de mano, por ahora limitada a pacientes con amputación de tipo desarticulación, lo que les permitiría conservar la funcionalidad de los músculos del antebrazo. 


\section{Metodología}

\section{A. Antropometría de la mano y parámetros de diseño}

\section{Antropometría de la mano}

Con el fin de garantizar el tamaño adecuado de la prótesis y sus componentes para una población mexicana, se estudiaron las medidas antropométricas de la población (Chaurand, León, \& Muñoz, 2007). Este estudio está basado en una población de 18 a 67 años de edad con datos de personas de la Ciudad de México y Guadalajara, todos ellos masculinos. La Tabla 2 muestra los valores promedio reportados y que servirán como base para el diseño de la prótesis.

Tabla 2. Medidas promedio de la población mexicana.

Realizada con datos de (Chaurand et al., 2007)

\begin{tabular}{|l|c|}
\hline \multicolumn{1}{|c|}{ Parámetros } & Valores reportados \\
\hline Largo de la mano & $181.41 \mathrm{~mm}$ \\
\hline Longitud de la palma de mano* & $103.51 \mathrm{~mm}$ \\
\hline Anchura de la mano sin pulgar & $82.55 \mathrm{~mm}$ \\
\hline Anchura de la mano con pulgar & $97.62 \mathrm{~mm}$ \\
\hline
\end{tabular}

Las dimensiones de las falanges se obtuvieron del trabajo publicado por investigadores de la UNAM (Hernández \& Alvarez, 2017) y de (Binvignat, Almagià, Lizana, \& Olave, 2012) quienes reportan las medidas de falanges obtenidas para mujeres y hombres de diferentes edades. Los valores reportados para hombres de entre 44 y 49 años de edad, mano izquierda, se presentan en la Tabla 3. Se eligieron estos valores ya que la prótesis, se plantea construir en primera instancia para un individuo de 48 años, masculino, de ocupación profesor de computación.

Tabla 3. Valores promedio y desviación estándar de longitudes de falanges de la mano adulta, con datos de (Binvignat et al., 2012).

\begin{tabular}{|c|c|c|c|}
\hline & \multicolumn{3}{|c|}{ Longitudes de falanges [mm] } \\
\hline & Falange Proximal & Falange Medial & Falange Distal \\
\hline Meñique & $37 \pm 10$ & $23 \pm 5$ & $20 \pm 3$ \\
\hline Anular & $45 \pm 11$ & $30 \pm 6$ & $23 \pm 3$ \\
\hline Medio & $57 \pm 13$ & $37 \pm 8$ & $26 \pm 2$ \\
\hline Índice & $50 \pm 11$ & $32 \pm 7$ & $25 \pm 3$ \\
\hline pulgar & $43 \pm 6$ & NA & $32 \pm 4$ \\
\hline
\end{tabular}




\section{Criterios de diseño}

Considerando que la prótesis debe cumplir con las dimensiones adecuadas para poder activar las teclas de un teclado de computadora ordinario, se investigó la distancia entre teclas. Se consideraron los teclados de computadoras portátiles de 17" y 15" que son las más comunes. Se consideraron especialmente aquellas computadoras con capacidades para ejecutar juegos de video, por ejemplo la MSI dominator G, Lenovo Y700 o Dell Alienware R5, etc. También se consideraron teclados de computadoras de escritorio, para ello se tomaron en cuenta las medidas de teclados estándar avalados por CherryMX, una de las empresas más importantes a nivel mundial en la manufactura de teclados.

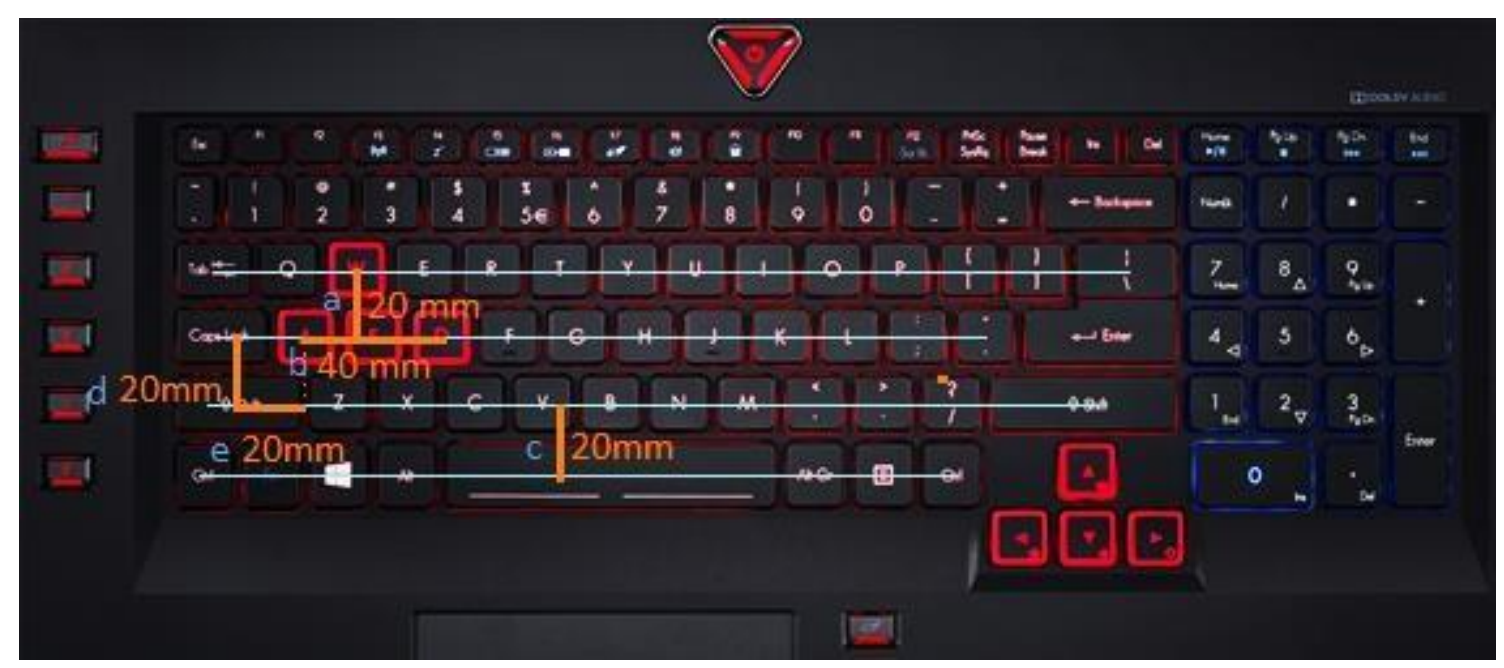

Figura 1. Dimensiones de separación entre teclas de ordenadores.

a) distancia entre línea de teclas ASD y línea QWERTY, b) separación entre teclas A-D en su punto central, c) separación entre línea CVB y tecla Space,

d) separación entre línea ASD y CVB.

Tomando en cuenta lo anterior, se determinó que se debe considerar una separación entre el centro de cada tecla con respecto a la tecla más cercana de $20 \pm 1 \mathrm{~mm}$ tanto horizontal como verticalmente (ver Figura 1). Por lo tanto, el diámetro y longitud de los dedos podría ajustarse un poco considerando estas dimensiones y las tareas principales de la prótesis, jugar o escribir.

\section{B. Diseño mecánico}

La mano humana se puede modelar mecánicamente como un sistema de 20 a 26 grados de libertad (DOF, por sus siglas en inglés), (Jaworski \& Karpiński, 2017), donde cada grado de libertad representa un tipo de movimiento de la articulación. Partiendo del trabajo realizado por (Cobos, Ferre, Sanchez Uran, Ortego, \& Pena, 2008) y considerando el diseño más simple posible para la interfaz con el teclado, se consideraron articulaciones rotacionales de 1 DOF 
para cada una de las falanges y toda la zona metacarpiana se consideró fija. Con base en lo anterior se determinó un modelo mecánico de 15 DOF, ver Figura 2. Donde L1, L2 y L3 representan las longitudes de las falanges de cada dedo, el resto de las medidas se fijaron como sigue: $L 4=103.51 \mathrm{~mm}, L 5=$ $93.51 \mathrm{~mm}, \mathrm{~L} 6=83.51 \mathrm{~mm}, \mathrm{~L} 7=43.50 \mathrm{~mm}, \mathrm{~L} 8=32.75 \mathrm{~mm}, \mathrm{~L} 9=24.66 \mathrm{~mm}$, $\mathrm{L} 10=31.94 \mathrm{~mm}, \mathrm{~L} 11=11.91 \mathrm{~mm}, \mathrm{~L} 12=9.34 \mathrm{~mm}, \mathrm{~L} 13=21.25 \mathrm{~mm}$.
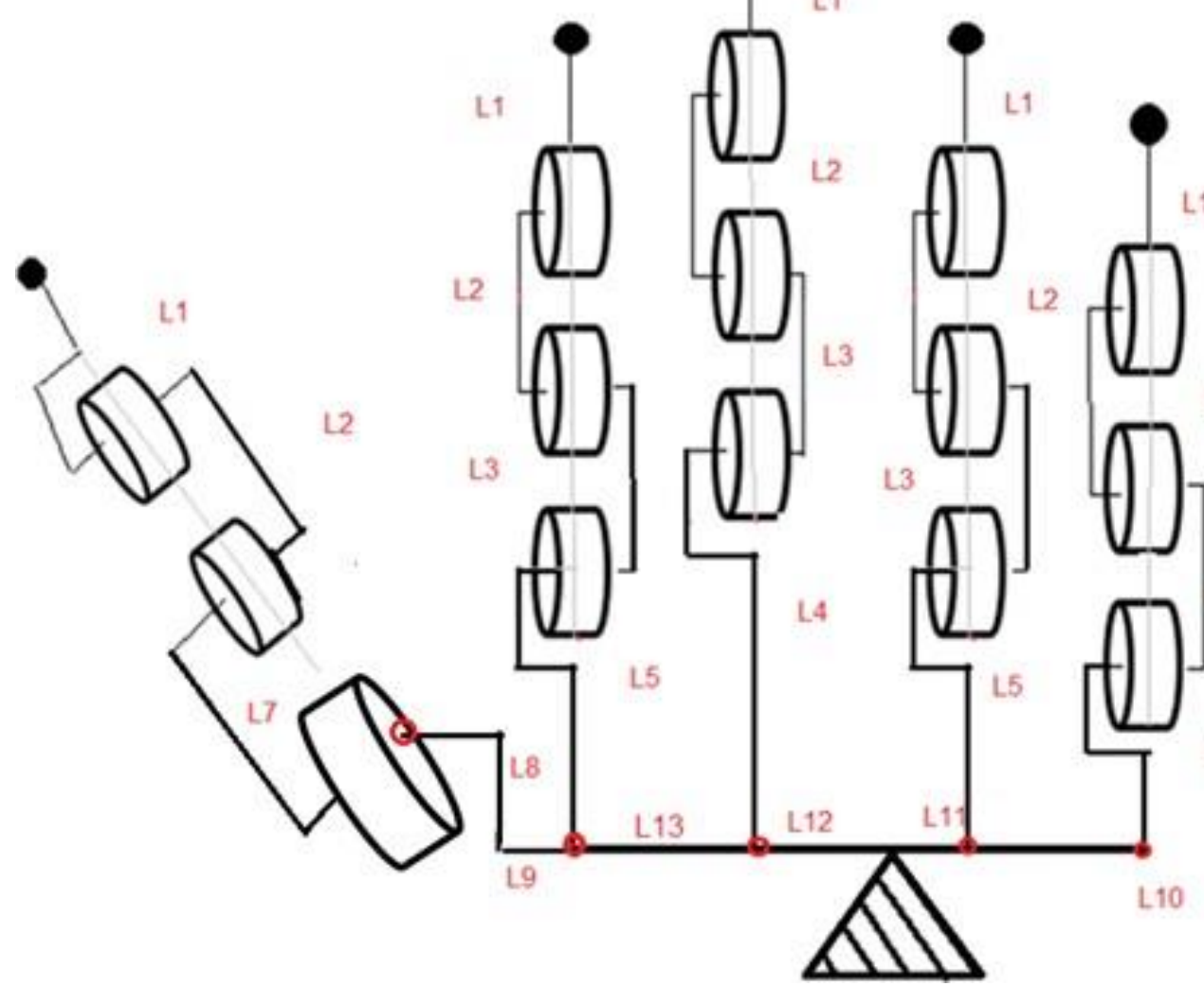

Figura 2. Diagrama de articulaciones de la prótesis de mano propuesta.

El ángulo de inclinación de la articulación CMC (carpometacarpiana) del pulgar se propuso en $45^{\circ}$ ya que es el ángulo promedio del dedo pulgar en reposo (Taboadela, 2007). Considerando que los metacarpianos se tomarían como un elemento único, sin movimiento, se diseñó la estructura de la palma de la mano como una pieza sólida. Las dimensiones de largo y ancho de la palma se basaron en los datos descritos en la sección de antropología de la mano, largo $(103.51 \mathrm{~mm})$ y ancho $(82.55 \mathrm{~mm})$. Para las falanges de los dedos se realizaron dos variantes en los diseños, una para las falanges del dedo meñique y otra variante para las falanges del dedo índice, medio y anular. Estas falanges cumplen con las medidas presentadas en la Tabla 3. 
Todas las falanges distales constan de dos piezas, una pieza que es la estructura de la falange y una que sirve como tapa o punta de los dedos que podría ser eventualmente reemplazada por un material compatible con pantallas touch o trackpads. Las falanges media y distal fueron diseñadas para presentar flexión únicamente en un sentido, por lo que no hay riesgo de que la pieza se flexione en dirección opuesta, mientras que, la falange proximal, al considerarse la dirección del sistema de transmisión de movimiento, no requirió esta medida, por lo que es la única pieza que puede ser ensamblada en cualquier sentido.

Todos los componentes se diseñaron utilizando el Software SolidWorks® 2018. En la Figura 3 se pueden observar cada una de las piezas diseñadas.
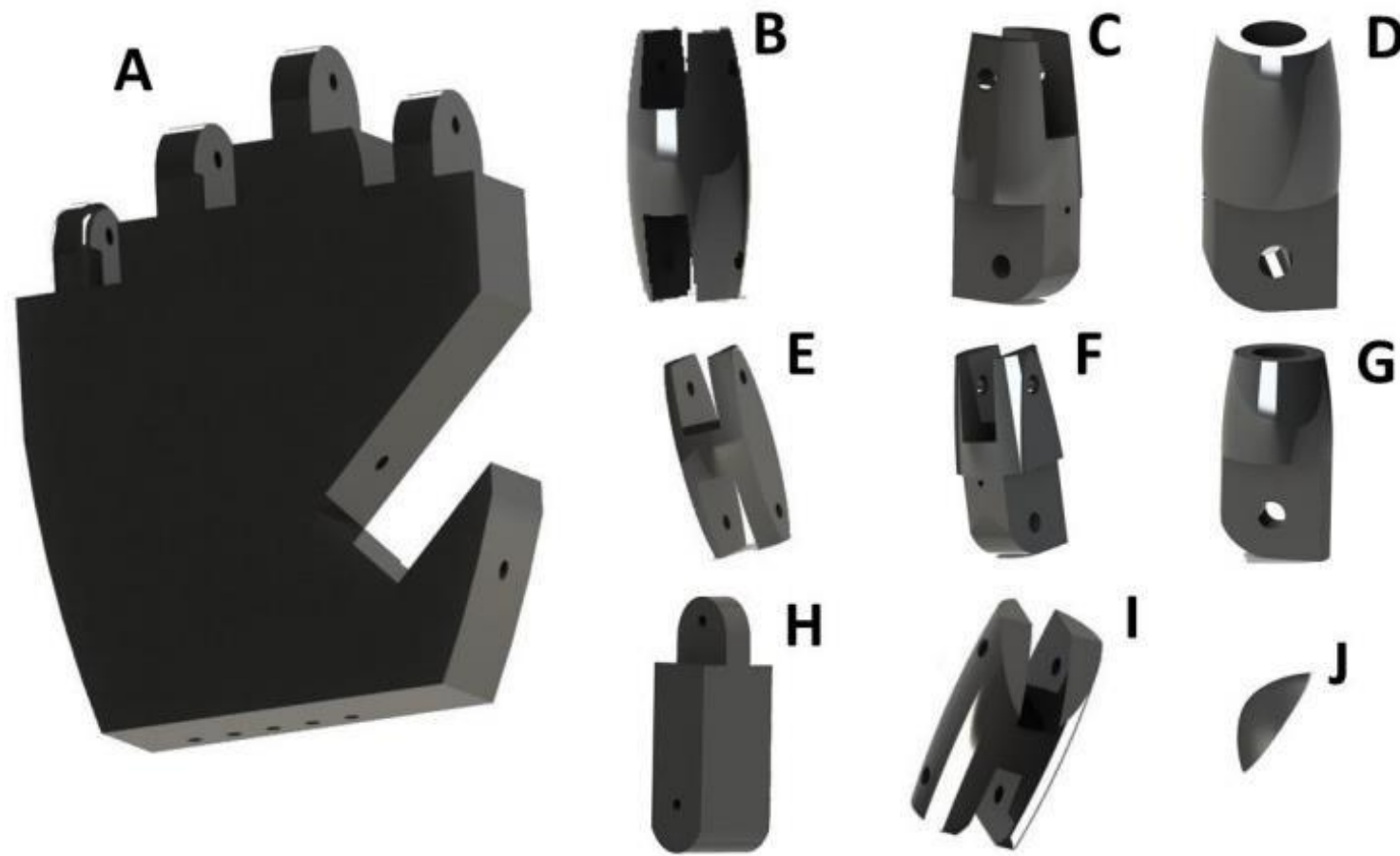

Figura 3. Piezas modeladas en 3D para cada segmento de la prótesis de mano. A) Palma de la mano, B) Falange proximal, C) Falange Medial, D) Falange distal, E) Falange proximal meñique, F) Falange medial meñique, G) Falange distal meñique, H) Metarcarpiano de pulgar, I) Falange pulgar, J) Punta de dedos.

En la Figura 4 se puede observar la renderización del modelo conceptual de la prótesis ya ensamblado. 


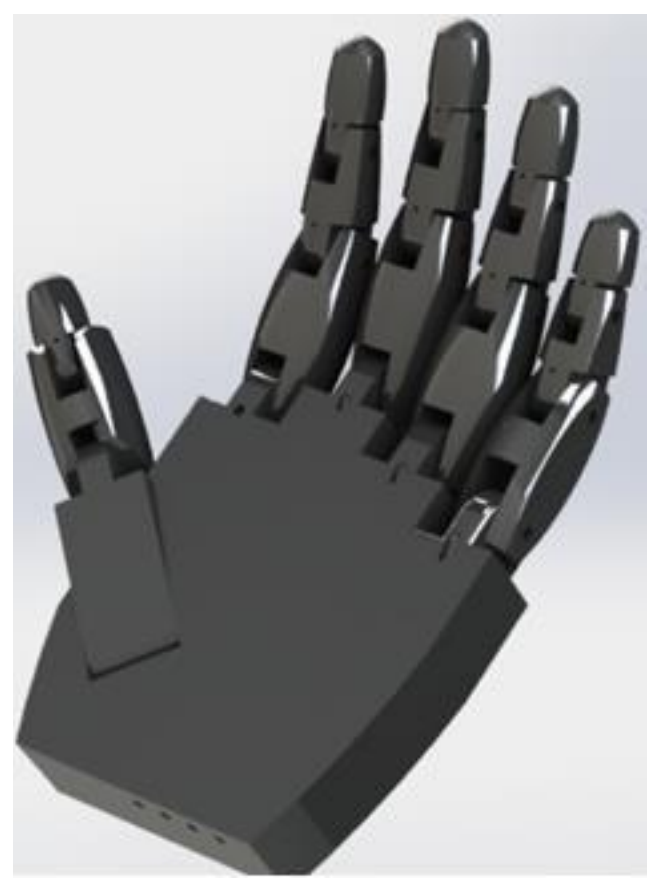

Figura 4. Diseño conceptual 3D de la mano protésica.

\section{Selección de materiales}

Considerando que la prótesis está diseñada para ser fabricada por medio de impresión 3D, se evaluaron los diferentes materiales de impresión, disponibles actualmente. Se consideraron los datos publicados por Ultimaker, una empresa de venta de equipo de impresoras 3D y suministros, y se evaluaron los termoplásticos que tienen en su catálogo tales como ácido poliláctico (PLA), polietileno clorado (CPE) y acrilonitrilo butadieno estireno (ABS). Así mismo, se compararon sus propiedades con las del titanio, material con el que construyen gran parte de las prótesis comerciales. En la Tabla 4 se muestran las principales propiedades de dichos materiales.

Tabla 4. Propiedades de los principales materiales de impresión 3D y de titanio. ND=Información no disponible.

\begin{tabular}{|l|l|l|l|l|}
\hline \multicolumn{1}{|c|}{ Característica } & \multicolumn{1}{c|}{ ABS } & \multicolumn{1}{c|}{ PLA } & \multicolumn{1}{c|}{ CPE } & \multicolumn{1}{c|}{ Titanio } \\
\hline Densidad $\left(\mathrm{g} / \mathrm{cm}^{3}\right)$ & 1.04 & 1.24 & 1.27 & 4.50 \\
\hline Esfuerzo de tracción a la ruptura & $33.9 \mathrm{MPa}$ & $45.6 \mathrm{MPa}$ & $37.7 \mathrm{MPa}$ & $220 \mathrm{MPa}$ \\
\hline Esfuerzo de tracción a la deformación & $39.0 \mathrm{MPa}$ & $49.5 \mathrm{MPa}$ & $41.1 \mathrm{MPa}$ & $140 \mathrm{MPa}$ \\
\hline Alargamiento a la deformación & $3.5 \%$ & $3.3 \%$ & $4.7 \%$ & $\mathrm{ND}$ \\
\hline Alargamiento a la rotura & $4.8 \%$ & $5.2 \%$ & $5.1 \%$ & $54 \%$ \\
\hline Resistencia a la flexión & $70.5 \mathrm{MPa}$ & $103 \mathrm{MPa}$ & $79.5 \mathrm{MPa}$ & $\mathrm{ND}$ \\
\hline Resistencia a la prueba de impacto Izod & $10.5 \mathrm{~kJ} / \mathrm{m}^{2}$ & $5.1 \mathrm{~kJ} / \mathrm{m}^{2}$ & $4.0 \mathrm{~kJ} / \mathrm{m}^{2}$ & $55.4 \mu \Omega / \mathrm{cm}$ \\
\hline Temperatura de fusión & $225^{\circ} \mathrm{C}$ & $145^{\circ} \mathrm{C}$ & $70^{\circ} \mathrm{C}$ & $1650^{\circ} \mathrm{C}$ \\
\hline Módulo de elasticidad & $1681.5 \mathrm{MPa}$ & $2345.5 \mathrm{MPa}$ & $1537.5 \mathrm{MPa}$ & $116 \mathrm{MPa}$ \\
\hline
\end{tabular}


Con base en el análisis de las propiedades de los distintos materiales, se determinó que el PLA resulta una mejor elección considerando sólo la resistencia a la flexión, comparado con el ABS y el CPE. Sin embargo, considerando que uno de los problemas de las prótesis actuales es su elevado peso, se debe elegir un material con la menor gravedad específica. Por lo que el ABS pudiera resultar la mejor opción.

\section{Transmisión de movimiento}

En la mano, los músculos que generan la flexión o extensión de los dedos, no forman parte del dedo, sino que se encuentran en el antebrazo y es por medio de tendones que se transmite el movimiento a los dedos. En el estado del arte, los métodos de transmisión de movimiento más comunes, así como sus principales ventajas y desventajas son:

- Transmisión de movimiento lineal por barras: Son precisos, económicos, control de velocidad de desplazamiento con respecto al punto de entrada. Sin embargo el diseño del mecanismo es complejo, el torque se reduce en el punto final, la resistencia de eslabones se compromete debido a la longitud del mismo.

- Actuadores en cada articulación: Se puede lograr un control preciso, capacidad de mimetizar los movimientos de la mano y se puede lograr una gran velocidad, sin embargo, añaden peso, incrementan costos, aumentan la complejidad del control, mayor consumo de energía.

- Transmisión de movimiento lineal por poleas fijas: Requieren un único actuador, reducen costos, peso, complejidad, aumento de torque en el elemento terminal con respecto al inicial, fáciles de controlar, sin embargo, reducen rango de movimiento, el sistema de transmisión se puede romper fácilmente y son poco precisos.

Considerando el objetivo de la prótesis propuesta, se considera como mejor opción la transmisión por poleas. Principalmente por su bajo costo, facilidad de control, reducción de peso y simplicidad de diseño. Se elige un sistema de guía por medio de canales dentro de la estructura de la prótesis, como se muestra en la Figura 5. 


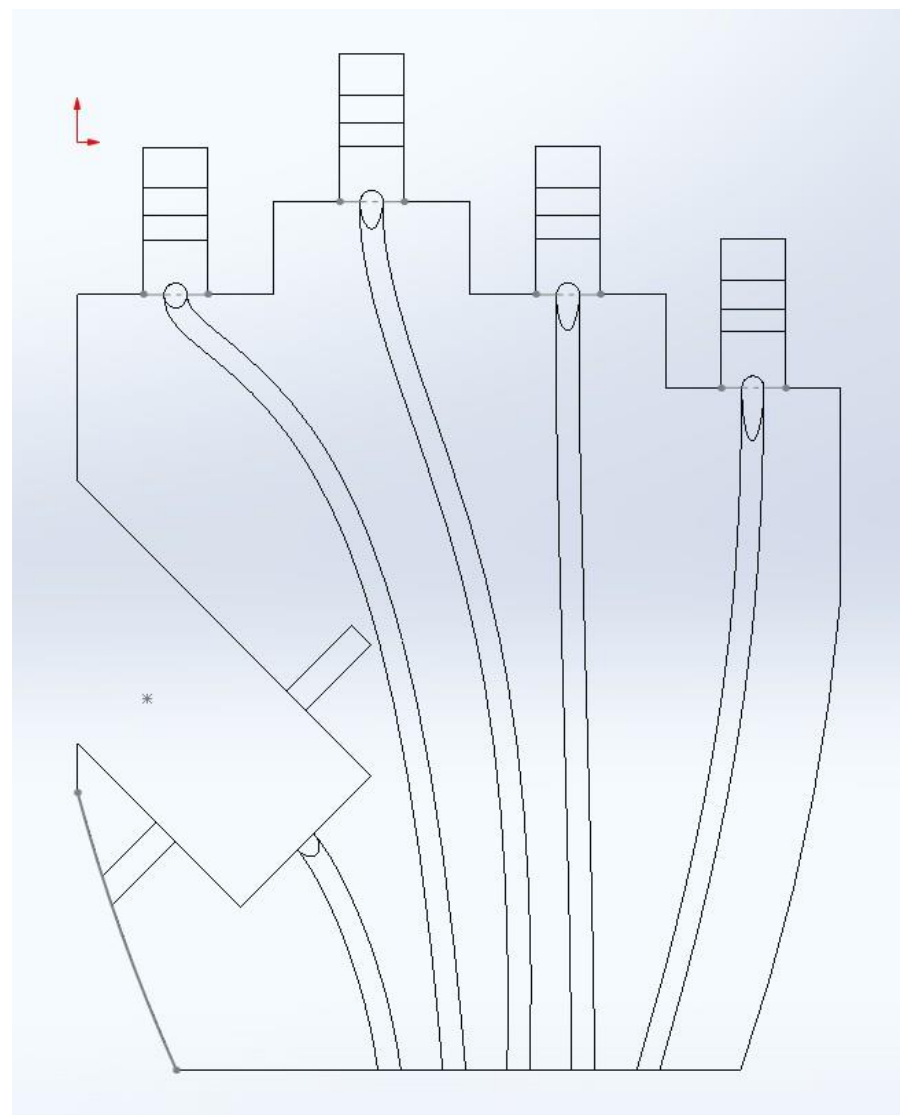

Figura 5. Canales guía de poleas de transmisión de movimiento.

Se plantea que cada polea tenga un actuador. El elemento transmisor se plantea que sea hilo de poliéster de $2.2 \mathrm{~mm}$ de grosor, el cual cuenta con una duración garantizada de 10 años y capaz de resistir tensiones de hasta $133 \mathrm{~kg}$ (Projar, 2018).

\section{Selección de actuadores}

La mayoría de teclados requieren de una fuerza de $0.58 \mathrm{~N}$ para activar una tecla, con un desplazamiento vertical de la tecla de $4 \mathrm{~mm}$ (Deskthority, 2014). Los teclados de membrana requieren entre 0.28 a $0.83 \mathrm{~N}$ (Shin, 2005). Considerando esto y que la transmisión por poleas no reduce demasiado el torque de entrada, se eligen servomotores que sean capaces de suministrar ese torque al menos, que sean los suficientemente pequeños como para acoplarse a la estructura de la mano sin incrementar demasiado el peso y que sean de bajo costo y bajo consumo de energía.

Después de revisar diversos servomotores fácilmente disponibles, se eligen los servomotores MG90s, los cuales pueden generar un torque de $1.8 \mathrm{~N}$, pesan $13.4 \mathrm{~g}$, miden $22.2 \times 11.8 \times 3.1 \mathrm{~mm}$, con una velocidad de 600 grados $/ \mathrm{s}$ y voltaje de alimentación de 4.8 a 5 V. Cada uno de estos actuadores será activado por las señales de control derivadas de las señales EMG de los músculos en el antebrazo. 


\section{Diseño del sistema de adquisición y procesamiento de las señales EMG}

\section{Determinación de número y colocación de electrodos.}

Con el fin de determinar el número y localización óptima de los electrodos para activar los actuadores, se ubicaron los músculos flexores de los dedos. Mediante el sistema de adquisición de EMG Trigno (Delsys. Inc, USA), se realizó la adquisición de la señal EMG en cada uno de los músculos, en diferentes posiciones, mientras se movían cada uno de los dedos, simulando el uso de un teclado. En la Figura 6 se muestra la colocación propuesta para cada par de electrodos.

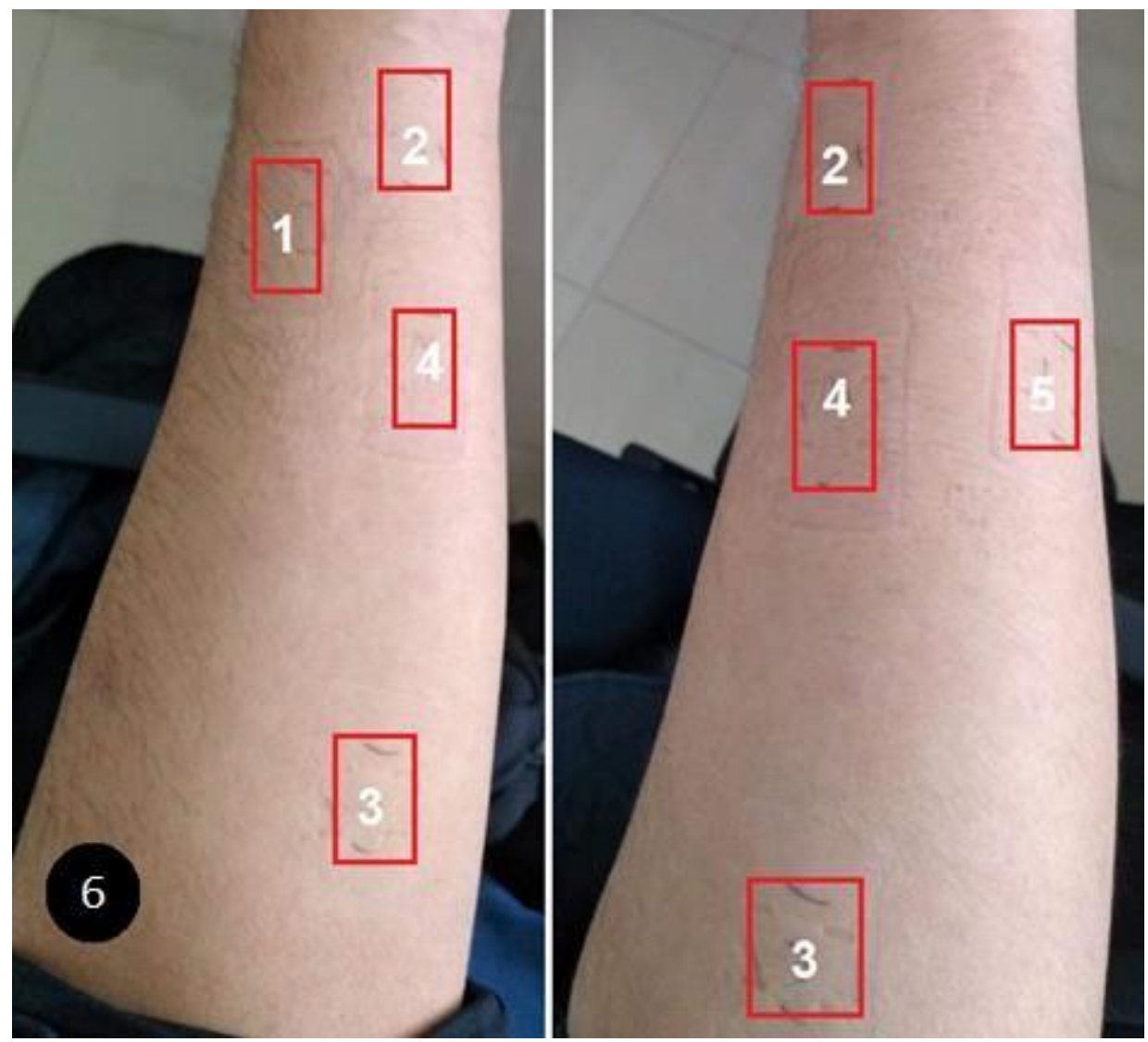

Figura 6. Localización de los electrodos en una vista anteroposterior del brazo izquierdo donde: 1 es el arreglo de electrodos para el pulgar; 2 el arreglo para el dedo índice; 3 el arreglo para el dedo medio; 4 el arreglo para el dedo anular; 5 el arreglo para el dedo meñique; 6 el electrodo de referencia.

Se propone un par de electrodos para cada punto, con una separación entre electrodos de $1.5 \mathrm{a} 2 \mathrm{~cm}$ y un electrodo de referencia para todos ellos. Los electrodos a utilizar podrían ser desechables de tipo $\mathrm{Ag}-\mathrm{AgCl}$. En general, la colocación de todos los electrodos proporciona una señal claramente relacionada con el dedo que se busca activar. Solamente la activación del dedo medio es el que pudiera ser difícil de discernir debido a que, por su posición, el resto de señales también presentan una amplitud apreciable. Sin embargo, aun 
así la señal rectificada y filtrada presenta una amplitud mayor al resto de las señales como se puede observar en la Figura 7. En dicha figura se muestran las señales de EMG captadas por los cinco sensores Trigno, colocados como se mostró en la Figura 6. Dichas señales se generaron cuando el sujeto de prueba realizó el movimiento únicamente del dedo medio únicamente. Se puede observar que las señales generadas por los músculos adyacentes tienen una amplitud menor a la señal captada para el dedo medio.

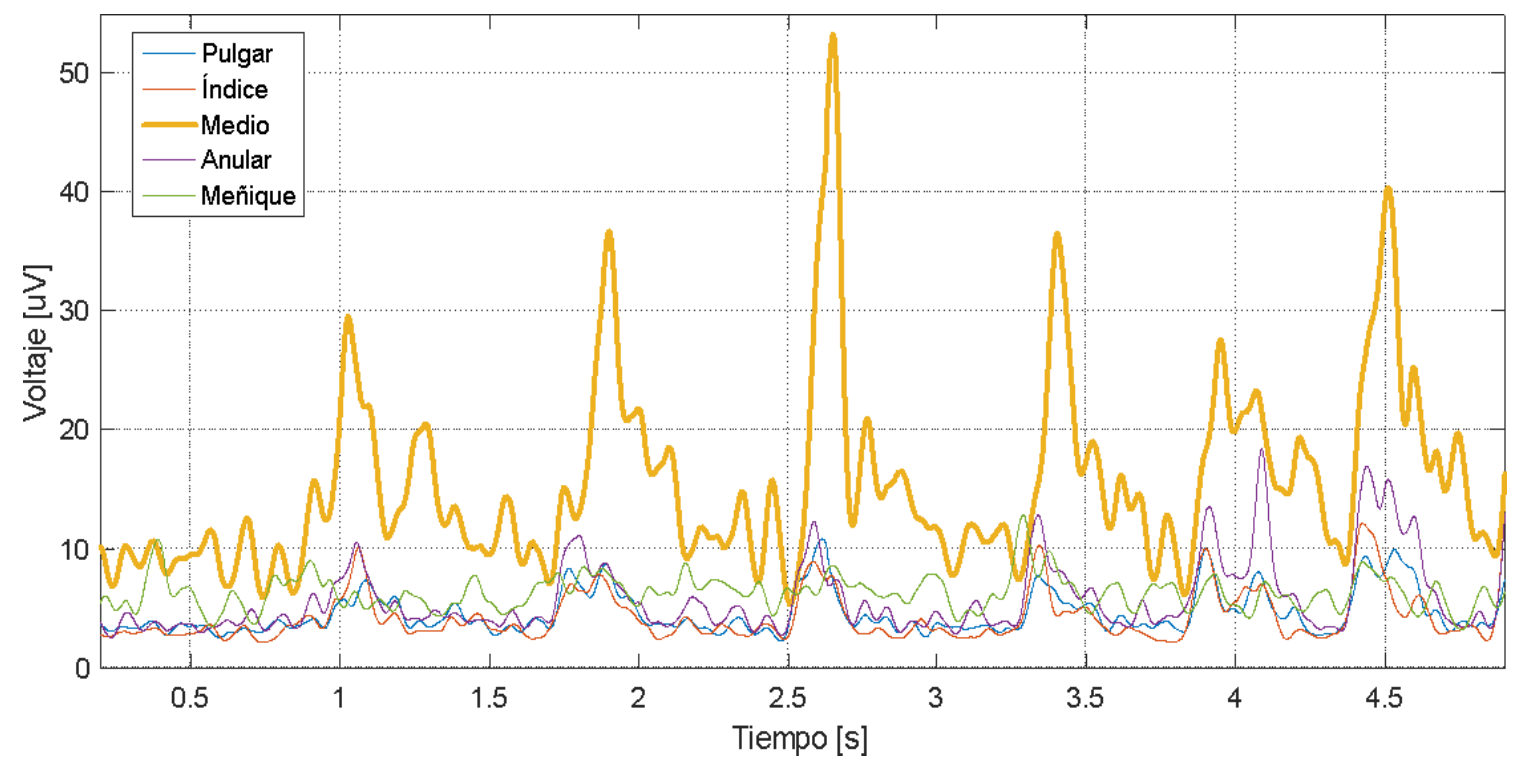

Figura 7. Señales de EMG durante la activación solamente del dedo medio.

El resto de señales, para la activación de cada uno de los otros dedos, presentan una amplitud mucho mayor, comparadas con el resto de señales por lo que, por medio de umbrales de activación, se puede determinar de manera simple, cual dedo se desea activar y enviar esa señal al controlador de los actuadores.

\section{Acondicionamiento y procesamiento de señal}

En el estado del arte existen un sinnúmero de circuitos electrónicos para la adquisición de señales electromiográficas. En este trabajo se propone un circuito de adquisición y acondicionamiento de señales muy simple pero, que cumple con las características necesarias para obtener las señales de EMG para la activación de los servomotores.

En la Figura 8 se muestra el diagrama del circuito electrónico. Este consta de un amplificador de instrumentación INA128 (Texas Instruments, USA), para adquirir la señal diferencial de los electrodos. Un OPA2604 para retroalimentar la señal al usuario, reduciendo así el nivel de ruido. Cuenta con un filtro 
pasabanda compuesto de un filtro pasa altas $(20 \mathrm{~Hz})$ y otro pasa bajas (452 $\mathrm{Hz}$ ) de segundo orden, implementados ambos con topologías Sallen-Key, con respuesta Butterworth.

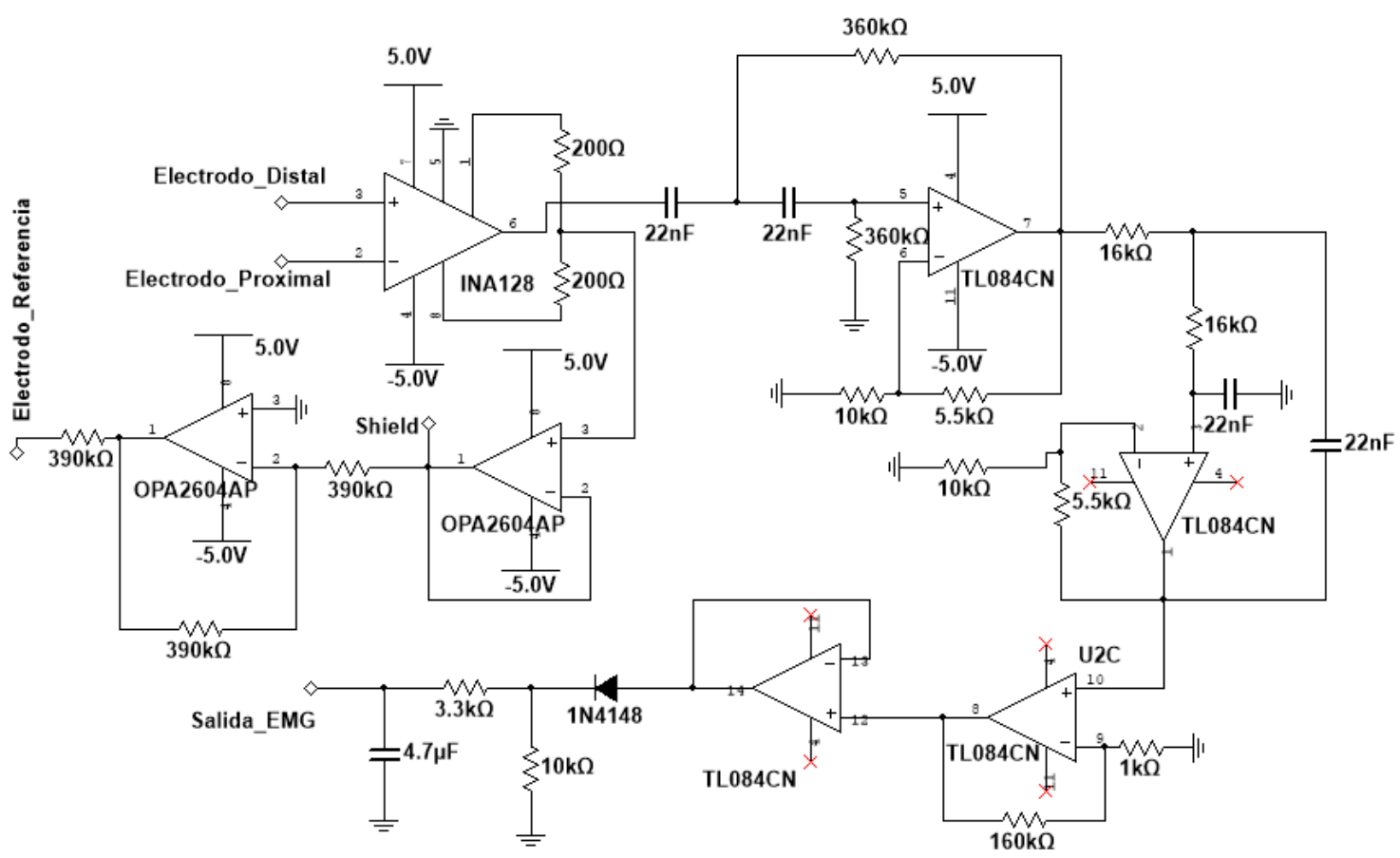

Figura 8. Circuito electrónico para la adquisición y acondicionamiento de señal EMGpor canal.

El filtro pasabanda (formado por el filtro pasabaja y pasa altas), además de limitar el ancho de banda de la señal, proporciona una amplificación de 2.4 veces la señal de entrada. Después de este filtro se conecta un amplificador no inversor, realizado con el OPAM TL084. Este amplificador proporciona una ganancia de 160 veces la entrada. Por lo que la ganancia total del circuito hasta este punto sería de 386.8 veces. Considerando ganancia dada por el amplificador de instrumentación (126 veces), la ganancia total es de 48,737 veces. Suficiente para incrementar los valores del orden de $50 \mu \mathrm{V}$ a $2.5 \mathrm{~V}$ aproximadamente. A esta señal amplificada y filtrada, se le aplica una rectificación implementada con un OPAM TL084 y un diodo 1N4148, el cual tienen un tiempo de recuperación de 4 ns y es apropiado para rectificar las señales del EMG. Finalmente, la señal rectificada es filtrada mediante un filtro pasivo pasabajas de primer orden, con el fin de suavizar la señal. El filtro está diseñado para cortar a una frecuencia cercana a $10 \mathrm{~Hz}$.

La señal acondicionada se digitaliza por medio de una tarjeta Arduino Mega®, el cual será el sistema que se encargará de activar los actuadores dependiendo de las señales de EMG. La Figura 9 muestra una propuesta de la conexión del sistema de control electrónico de la mano protésica. Se puede observar que la 
tarjeta Arduino se encargará de muestrear las señales acondicionadas de EMG y activar los actuadores cuando detecte una señal de EMG propia de la activación de cada dedo.

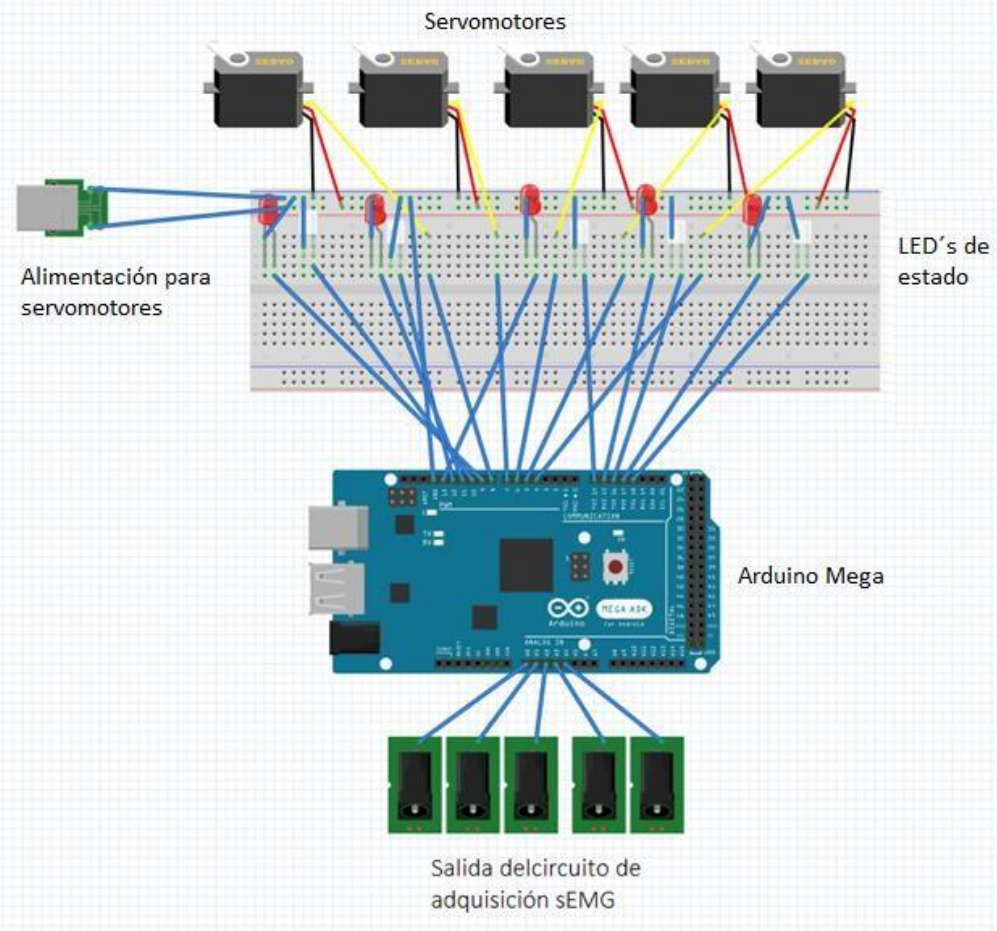

Figura 9. Conexión de servomotores, circuitos de adquisición de EMG y Arduino Mega.

En la Figura 10 se muestra el diagrama de flujo del firmware que podría controlar la activación de los dedos de la mano protésica propuesta. El funcionamiento de este se basa en la lectura de canales de EMG, detección por umbrales y activación de servomotores para poder presionar las teclas.

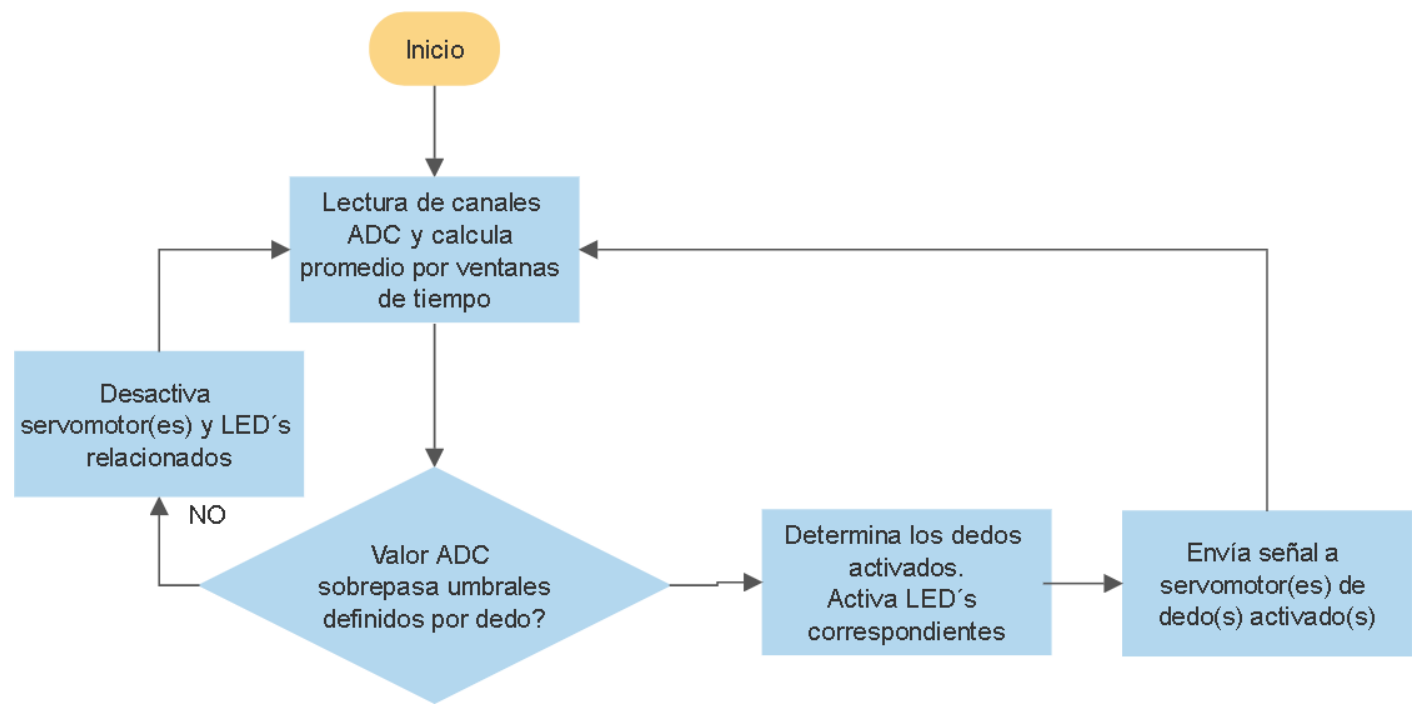

Figura 10. Diagrama de flujo de firmware en el microcontrolador del Arduino para activar la mano protésica. 


\section{Resultados}

\section{Sobre el diseño mecánico y selección de materiales}

Se propuso el diseño de una prótesis de mano de 15 DOF, que se considera como la configuración más simple que permitiría la activación de cada uno de los cinco dedos de una manera similar a una mano biológica. La prótesis propuesta consta de 14 piezas diseñadas en SolidWorks, las cuales pueden ser fácilmente imprimibles usando impresoras $3 D$ de bajo costo. El material seleccionado podría ser cualquiera de los más comunes, PLA o ABS. Ambos tienen la capacidad de soportar las fuerzas requeridas para presionar una tecla $(0.58 \mathrm{~N})$. Se propone el uso de ABS debido a su menor densidad, lo que implicaría un menor peso. Un cálculo en el software SolidWorks, arroja una estimación 191 gramos de material para la impresión de la prótesis. Agregando los cinco servomotores $(13.4 \mathrm{~g} / \mathrm{cu})$, se obtendría una mano con un peso aproximado de $258 \mathrm{~g}$. Considerando el peso de la tarjeta Arduino Mega (36 g) y baterías recargables y las tarjetas de acondicionamiento de señal, se estima que la mano pesaría menos de $500 \mathrm{~g}$. Esto es 3 veces menos que la prótesis comercial i-limb de quantum, por mencionar alguna.

Por otro lado, dado que el diseño considera que con una rotación de $90^{\circ}$ se obtendrá el recorrido total del dedo y, tomando en cuenta la velocidad de $600 \%$ del servomotor elegido, se consigue una velocidad de $150 \mathrm{~ms}$ para el recorrido total del dedo. Considerando que el dedo regrese a su posición original en lapso similar de tiempo, se podría realizar un ciclo de pulsación en $300 \mathrm{~ms}$ aproximadamente. Esto significa poco más de 3 PPS (pulsaciones por segundo) o 180 PPM (pulsaciones por minuto), siempre y cuando se pulsara la misma tecla. Se estima que un mecanógrafo experto puede realizar entre 42 y 54 PPM (Testdemecanografía, 2018). Por lo tanto, la prótesis podría ser capaz de alcanzar esa velocidad si el usuario practica lo suficiente. La precisión de pulsación dependerá de la destreza del usuario para posicionar la mano moviendo el antebrazo y su control de músculos para la activación de los servomotores.

\section{E. Sobre la adquisición y acondicionamiento de señal}

Se realizó el diseño del circuito electrónico para la adquisición de las señales electromiográficas. El diseño está basado en el propuesto por el fabricante del amplificador de instrumentación INA128 (Texas Instruments) y en circuitos de filtrado y rectificación de uso común (Wang, Tang, \& E Bronlund, 2013). Se calculó una ganancia del circuito lo suficientemente alta como para llevar las señales de entrada de los electrodos a un rango entre 2 y $3 \mathrm{~V}$ como máximo para evitar saturar el ADC de la tarjeta Arduino. Se consideraron las magnitudes de la señal obtenidas de los experimentos usando el sistema 
Trigno (Delsys.inc) descrito en la sección $\mathrm{C}$ de este documento. Una simulación solamente de la sección de filtrado y amplificación secundaria se puede observar en la Figura 11. Las amplitudes de la señal EMG observadas en las posiciones elegidas estuvieron alrededor de $50 \mathrm{uV}$ pico, ya filtradas y rectificadas. La ganancia total del circuito electrónico, considerando la ganancia del INA128, es de alrededor de 48,737 veces la señal de entrada.

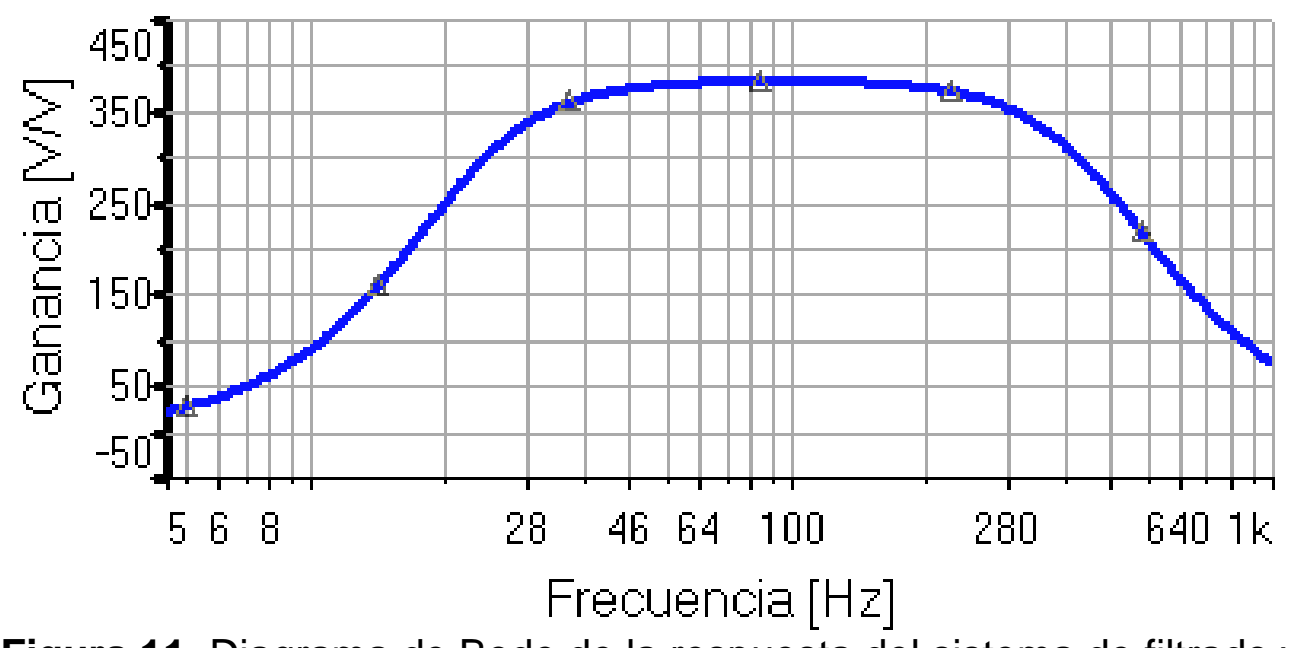

Figura 11. Diagrama de Bode de la respuesta del sistema de filtrado y amplificación propuesto para el circuito de adquisición de señales EMG.

Con respecto al procesamiento de señales de EMG para determinar la intención de movimiento, existen muchas técnicas para el análisis, procesamiento y clasificación de las señales de EMG. Se pueden citar por ejemplo, técnicas usando inteligencia artificial basadas en redes neuronales, lógica difusa, modelos autoregresivos, wavelets y otras técnicas basadas en análisis de tiempo-frecuencia, como la distribución de Weiner-Ville (WVD) o la distribución de Choi-Williams, etc. Una descripción más completa de estas y otras técnicas se puede encontrar en (Chowdhury et al., 2013; Reaz, Hussain, \& Mohd-Yasin, 2006). Sin embargo, todas éstas técnicas tienen la desventaja de requerir análisis u operaciones complejas que difícilmente se pueden llevar a cabo en un microcontrolador o un sistema embebido de bajo costo. Debido a lo anterior se propuso el uso de un método simple basado en umbrales de las señales rectificadas. Los estudios preliminares de análisis de la señales EMG adquiridas usando el sistema Trigno (Delsys.inc), ver sección C, permiten indicar que ésta solución podría dar bueno resultados, sin embargo estos resultados dependerán del usuario de la prótesis y la calidad de las señales que se puedan adquirir de él. 


\section{Conclusiones}

En este trabajo se presenta el diseño de una mano protésica para facilitar el uso de teclados a usuarios con amputación de la mano izquierda, aunque este diseño puede ser fácilmente modificado para alguien con amputación en la mano derecha. La prótesis propuesta está diseñada para fabricarse por medio de impresión 3D y controlarse por medio de señales EMG. Se estima que la prótesis no pesará más de $500 \mathrm{~g}$, con un costo (incluyendo material y construcción a nivel artesanal o prototipo) inferior a los 300 USD.

Se propuso un sistema de adquisición y procesamiento de la señal de EMG lo más sencillo posible con la premisa de que éste pueda ser implementado en un sistema embebido de bajo costo, como lo es una tarjeta Arduino. La selección de componentes y materiales se llevó a cabo considerando los datos encontrados en el estado del arte y en las características de diseño fijadas inicialmente. Lo anterior permite inferir que el diseño es funcional, sin embargo, hasta que el sistema no se construya e implemente, no se tiene la certeza del funcionamiento $y$ es probable que se tengan que hacer adecuaciones dependiendo del usuario para el cual se diseñe la prótesis.

La principal aportación del diseño propuesto, además de su bajo costo, es el hecho de que está diseñado para cubrir un problema específico que hasta ahora, ninguna de las prótesis comerciales o reportadas en prototipos ha solucionado. La interfaz entre el usuario amputado y un teclado, debe ser lo suficientemente rápida y ligera como para garantizar un uso más natural del teclado. Esta aportación es especialmente interesante para personas que han sufrido amputaciones y que debido a ello buscan cambiar de empleo a alguno relacionado con las computadoras. O simplemente para disminuir su estrés por medio del uso de juegos de video.

\section{REFERENCIAS}

Amputee Coalition (2016). Limb Loss Statistics. Recuperado el 10 de mayo de 2019, de Amputee Coalition website: https://www.amputeecoalition.org/resources/limb-loss-statistics/

Bebionic. (2012). Technical Information - bebionic. Recuperado el 23 de abril de 2019, de http://bebionic.com/the_hand/technical_information/

Binvignat, O., Almagià, A., Lizana, P., \& Olave, E. (2012). Aspectos Biométricos de la Mano de Individuos Chilenos. International Journal of Morphology, 30(2), 599-606. https://doi.org/10.4067/S0717-95022012000200040

Chaurand, R. Á., León, L. R. P., \& Muñoz, E. L. G. (2007). Dimensiones antropométricas de población latinoamericana. 283. 
Chowdhury, R., Reaz, M., Ali, M., Bakar, A., Chellappan, K., \& Chang, T. (2013). Surface Electromyography Signal Processing and Classification Techniques. Sensors, 13(9), 12431-12466. https://doi.org/10.3390/s130912431

CONACyT. (2018). EI CIMAV diseña prótesis robótica. Recuperado el 23 de abril de 2019, de https://centrosconacyt.mx/objeto/el-cimav-disenaprotesis-robotica/

Deskthority. (2014). Force. Recuperado el 3 de mayo de 2019, de https://deskthority.net/wiki/Force

Hernández, A. G., \& Alvarez, M. del P. M. (2017). Arte y Ciencia: Proporción de los dedos de la mano. Latin-American Journal of Physics Education, 11(2), 28.

ISHN. (2014). Statistics on hand and arm loss. Recuperado el 10 de mayo de 2019, de Industrial Safety \& Hygiene News website: https://www.ishn.com/articles/97844-statistics-on-hand-and-arm-loss

Jason, M. (2017). Amputee Controls Individual Prosthetic Fingers. Recuperado el 11 de mayo de 2019, de Georgia Tech, Science and Technology website: https://music.gatech.edu/news/force-strong-amputee-controlsindividual-prosthetic-fingers

Jaworski, Ł., \& Karpiński, R. (2017). Biomechanics of the human hand. 3(1), 6.

Kate, J. ten, Smit, G., \& Breedveld, P. (2017). 3D-printed upper limb prostheses: a review. Disability and Rehabilitation: Assistive Technology, 12(3), 300-314. https://doi.org/10.1080/17483107.2016.1253117

LeBlanc, M. (2011). "Give Hope - Give a Hand" - The LN-4 Prosthetic Hand. Recuperado de https://web.stanford.edu/class/engr110/2011/LeBlanc03a.pdf

Mobius Bionics. (2018). LUKE Arm. Recuperado el 23 de abril de 2019, de Mobius Bionics website: http://www.mobiusbionics.com/luke-arm/

Open bionics. (2018). Hero Arm - an affordable, advanced and intuitive bionic arm. Recuperado el 11 de mayo de 2019, de https://openbionics.com/hero-arm/

Ottobock. (2019). Michelangelo Hand. Recuperado el 23 de abril de 2019, de https://shop.ottobock.us/Prosthetics/Upper-LimbProsthetics/Michelangelo-Axon-Bus-System/Michelangelo-HandAxonHook/Michelangelo-Hand-Transcarpal/p/8E550 5R-M

Perez-Rodriguez, A. (2017). Proyecto MyoArm: Prótesis robótica con sensado EMG y entrenamiento de redes neuronales. Universidad de Sevilla, Sevilla, España. 
Prensilia s.r.I. (2017). IH2 Azzurra. Recuperado el 11 de mayo de 2019, de Prensilia. grasping innovation website: https://www.prensilia.com/portfolio/ih2-azzurra/

Probionics. (2015). Probionics. Recuperado el 1 de septiembre de 2018, de http://www.probionics.com.mx

Projar. (2018). Hilo de Poliéster. Recuperado el 3 de mayo de 2019, de PROJAR website: https://www.projar.es/productos/productoshortofruticultura-jardineria/vina-y-cultivos-agricolas/hilo-de-poliester/

Reaz, M. B. I., Hussain, M. S., \& Mohd-Yasin, F. (2006). Techniques of EMG signal analysis: detection, processing, classification and applications. Biological Procedures Online, 8(1), 11-35. https://doi.org/10.1251/bpo115

Secretaría del Trabajo y Previsión Social. (2010). Información sobre acidentes y enfermedades de trabajo. Recuperado de http://www.stps.gob.mx/bp/secciones/dgsst/estadisticas/nacional\%20200 1-2010.pdf

Shin, D. (2005). Performance and Usability of Flexible Membrane Keyboards.

Taboadela, C. H. (2007). Goniometría : una herramienta para la evaluación de las incapacidades laborales (1a ed., Vol. 1). Recuperado de http://bibliomedicinadigital.fmed.uba.ar/medicina/cgibin/library.cgi?a=d\&c=catalogo\&d=CatalogoGral_00259

Testdemecanografía. (2018). Promedio de Palabras por Minuto y cómo mejorarlo. Recuperado el 11 de mayo de 2019, de Testsdemecanografía website: http://www.testdemecanografia.com/promedio-ppm/

Touch Bionics. (2015). i-limb quantum. Recuperado el 23 de abril de 2019, de http://touchbionics.com/products/active-prostheses/i-limb-quantum

Vanguardia. (2018). El salario promedio en México. Recuperado el 23 de abril de 2019, de https://vanguardia.com.mx/articulo/6-mil-117-pesos-al-mesel-salario-promedio-en-mexico

Vazquez-Vela, E. (2015, marzo 4). Los amputados, un reto para el estado. Recuperado de http://www.anmm.org.mx/actas2015/Acta_SO04032015.pdf

Wang, J., Tang, L., \& E Bronlund, J. (2013). Surface EMG Signal Amplification and Filtering. International Journal of Computer Applications, 82(1), 1522. https://doi.org/10.5120/14079-2073

Youbionic. (2014). Youbionic. Recuperado el 23 de abril de 2019, de Youbionic website: https://www.youbionic.com/ 


\section{Notas biográficas}

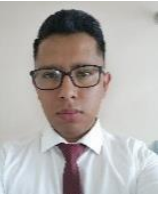

Ernesto-Eduardo López-López graduado de la Facultad de Medicina de la Universidad Autónoma del Estado de México como Bioingeniero Médico en 2019. Sus intereses de investigación están relacionados con el desarrollo de prótesis para rehabilitación y la robótica médica. Actualmente se desempeña como ingeniero de servicio biomédico.

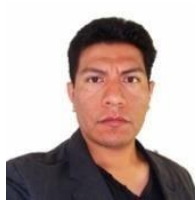

Rigoberto Martínez Méndez recibió el título de ingeniero en electrónica del Instituto Tecnológico de Apizaco, la maestría en bioelectrónica del CINVESTAV-IPN y el Doctorado en Biomédica de la Universidad de Chiba en Japón. Actualmente es profesor investigador en la Facultad de Ingeniería de la Universidad Autónoma del Estado de México. Sus principales intereses de investigación son la estimulación eléctrica, el diseño y desarrollo de dispositivos médicos no invasivos para diagnóstico y rehabilitación.

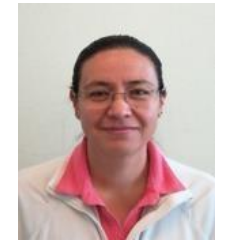

Adriana H. Vilchis González Obtuvo el grado de doctor en Imagen, Visión y Robótica en el Instituto Nacional Politécnico de Grenoble, Francia. Actualmente es profesor investigador en las Facultades de Ingeniería y de Medicina de la Universidad Autónoma del Estado de México. Sus intereses científicos incluyen Robótica Médica y Sistemas Mecatrónicos. Tiene 2 patentes internacionales en el área de Robótica Médica así como varios diseños industriales otorgados y patentes solicitadas ante el IMPI.

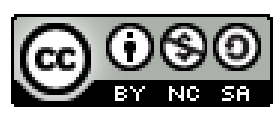

Esta obra está bajo una licencia de Creative Commons Reconocimiento-NoComercial-Compartirlgual 2.5 México. 